\title{
Numerical and Experimental Studies of Surface-pulsed Magneto-oscillation on Solidification
}

Jing Zhao ${ }^{\text {a, b }}$, Yufeng Cheng ${ }^{\text {a }}$, Ke Han ${ }^{\mathrm{c}}$, Xunzhe Zhang ${ }^{\mathrm{a}}$, Zhishuai Xu ${ }^{\mathrm{a}}$, Qijie Zhai ${ }^{\mathrm{a},}$ *

${ }^{a}$ Shanghai Key Laboratory of Modern Metallurgy \& Materials Processing, Shanghai University, Shanghai, 200072, P.R. China

${ }^{\mathrm{b}}$ Shanghai Institute of Materials Genome, Shanghai, China

${ }^{\mathrm{c}}$ Tangshan College, Hebei, 063020, P.R. China

${ }^{d}$ National High Magnetic Field Laboratory, Florida State University,

Tallahassee, 32310, USA

ABSTRACT: Both experimental and numerical methods were used to study the distribution of magnetic field, flow field, temperature, and solidification structure in pure Al under surface-pulsed magneto-oscillation (SPMO). The numerical modeling showed that electromagnetic force changed with time and location in molten Al. In the melt, electromagnetic force resulted in cyclic tensile and compressive loads and in intensive forced-convection. Experimental results demonstrated that application of SPMO refined the solidified structure and reoriented the growth direction of columnar grains. Results of both simulation and experimentation led to the conclusion that surface oscillations induced by electromagnetic force resulted in grain refinement while the corresponding forced convection contributed to change of growth direction 
Corresponding author. Tel.: +86 21 56331218; E-mail address: qjzhai@ shu.edu.cn (Qijie, Zhai)

of columnar grains.

KEYWORDS: Surface-pulsed magneto-oscillation, Simulation, Electromagnetic force, Convection, Refinement, Growth direction.

\section{Introduction}

The electric current pulse (ECP) and pulsed magnetic field (PMF) have been attracting more and more attention because they are techniques that are convenient to use and relatively safe for the environment. Liao et al. (2007) studied the effect of ECP on the solidified structure of Al. One disadvantage of the ECP technology is that the electric pulse must be directly passed through the metal melt. Special attention must be focused on production safety and the melt is easy to be contaminated. Gao et al. (2007) found that PMF induced by the electric current in the coil has to reach extremely high value in order to obtain satisfactory effect. As a result, undesirable splashing can occur at the top surface of the melt. In order to make improvement, Gong et al. (2008) developed a new technology named surface pulsed magneto-oscillation (SPMO), in which a cookie-like induction coil was employed. Yin et al. (2012) found that remarkable structural refinement was achievable with SPMO on Al melt. Refinement occurs with SPMO because the application of electromagnetic force causes crystal rain to form the top surface or mold wall. SPMO technology has many advantages. It is easy to use, and the coil 
touches neither the melt nor the mold. As a result, SPMO may have high potential for industry application. However, neither Gong et al.(2008) nor Yin et al. (2012) were able to clarify the variation of electromagnetic force and the flow field distribution during the whole period of application because of its transient, variable, and complex process. Fortunately, numerical simulation, which has never done on SPMO before, can provide effective analysis for experimentation and production. Many works on simulation of electromagnetic fields applied in material solidification process have been carried out. Kermanpur et al. (2011) developed a couple-field electromagnetic-thermal simulation model to design suitable induction coils for the electromagnetic levitation melting of metals. Barman et al. (2009) performed numerical and experimental studies on transport phenomena during solidification of an aluminum alloy in the presence of linear electromagnetic stirring. The results showed that the numerical predictions of temperature variations were in good agreement with experiments, and flow field evolution correlated well with resulting microstructure. Kolesnichenko et al. (1994) simulated electromagnetic force and flow distribution under PMF, and the variation in the pulse applying period was investigated. Le et al. (2007) simulated the flow pattern and temperature field of direct chill (DC) casting of magnesium alloys. They found that the lower temperature gradient and the shoaled liquid sump resulted from forced convection after applying Electronic Magnetic (EM) field. Ha et al. (2003) conducted a numerical study of the magnetic field effect on turbulent flow field, 
heat transfer, and solidification and confirmed the capability to help stable shell growth of steel slabs of electromagnetic brake. Li et al. (2011) simulated electromagnetic force and flow distribution in nickel-based super alloy melt with low voltage pulsed magnetic field (LVPMF) using commercial ANSYS finite element software. Ma et al. (2009) investigated the distribution of magnetic force, flow field distribution, and Joule heat under pulsed magnetic field (PMF). A numerical simulation was carried out, showing that the magnetic force in axial direction produced the convection of the melt and the magnetic force in radial direction produced the vibration of the melt.

It is apparent that application of an electromagnetic field affects the solidification process in a range of temperature and flow field variation. In this work, electromagnetic field, fluid flow, and temperature distribution in $\mathrm{Al}$ melt under SPMO were investigated with respect to the refinement effect of SPMO on the $\mathrm{Al}$ solidification structure.

\section{Numerical Models}

2.1 Model and boundary conditions

The SPMO casting setup shown in Fig. 1a consisted of an SPMO coil, a mold and the melt. The Finite Element Method (FEM) model shown in Fig. 1b was built on this setup. PLANE13 (from ANSYS $^{\odot}$ ), which is a computational code for calculation of electromagnetic field, flow field, and temperature field, was used in the calculation. Different grid sizes were chosen for different portion of the model. The finest grid 
was used in melt. The simulating procedure was divided into three steps: First, the

RLC (where R, L and C stand for electrical resistance, induction, and capacity)

electric circuit was solved and then substituted into the electromagnetic field model.

Second, the fluid flow model was solved with the acquired electromagnetic force in the electromagnetic field model. Finally, the temperature field was solved with the acquired Joule heat predicted by the electromagnetic field model.

The thermal physical parameters of the melt and mold are known to change with temperature. The main parameters of the pulse current used in the simulation include peak value and discharging frequency of electric pulse. Typical value are $100 \mathrm{~h}_{\mathrm{I}} \mathrm{A}$ and $2 k_{t} H z$, where $h_{I}$ and $k_{t}$ are the coefficients of the pulse generator. These values generally remain constant in the circuit.

The electrical resistivity was assumed to be $2.65 \times 10^{-7}$ (Huang et al, 2008) and $1.72 \times 10^{-8} \Omega . \mathrm{m}$ (Paul, 2004) for the aluminum melt and the coil, respectively. The density and viscosity of the fluid were assumed to be $2.385 \mathrm{~g} . \mathrm{cm}^{-3}$ and $2.51 \mathrm{mPa} . \mathrm{s}$, respectively (Zhang et al., 2007).
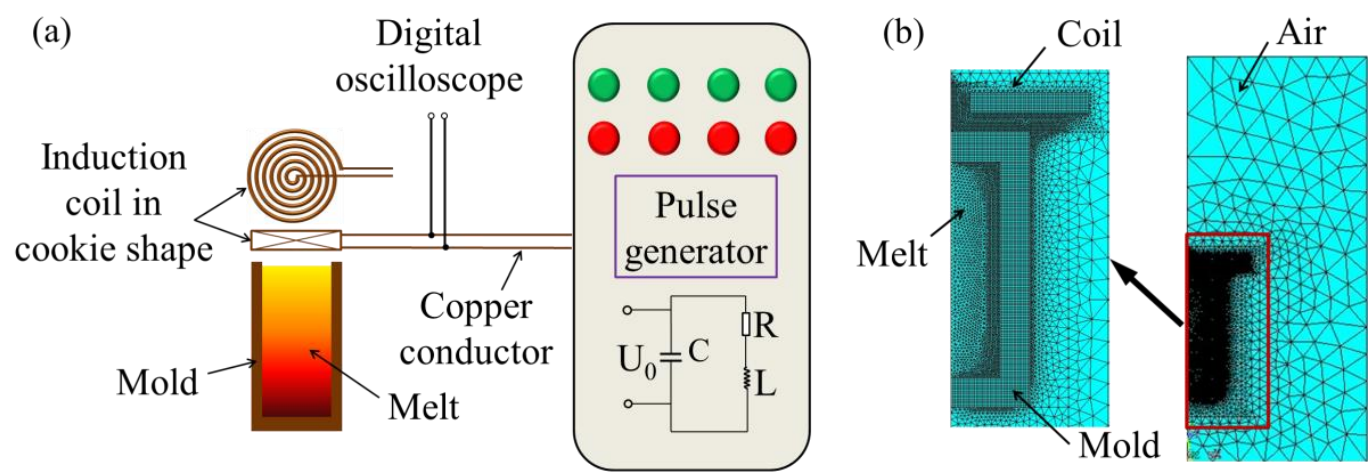

Figure 1. Schematic of SPMO casting setup (a) and FEM model used in the 
simulation (b).

\subsection{Governing equations}

To simplify mathematical model and save calculation time, the calculation was based on following assumptions: (1) The change of density of Al melt is smaller and can be neglected, so Al melt used in simulation was considered as a incompressible conductor; (2) The coil could be considered as current carrier with a smaller diameter, in which current density remained uniform throughout the coil; (3) No deformation occurred in the melt under magnetic force; (4) The system was cylindrical and symmetric and could thus be rendered as a two-dimensional model for simulation. Without above assumptions, more complicate model and more grids should be built and more calculation time would be expended.

The governing Maxwell equations of electromagnetic field can be expressed by:

$$
\begin{aligned}
& \nabla \times E=-\frac{\partial B}{\partial t} \\
& \nabla \cdot B=0 \\
& \nabla \cdot D=\rho_{0} \\
& \nabla \times H=J+\partial D / \partial t
\end{aligned}
$$

where $E$ is magnetic flux density vector, $B$ is magnetic flux density vector, $D$ is electric flux density vector, $\rho_{0}$ is electric charge density, $H$ is magnetic field intensity vector and $J$ is total current density vector.

The constitutive relations for the related electric fields are described in the following equations: 
$J=\sigma(E+v \times B)$

$D=\varepsilon E$

$B=\mu_{0} H$

where $\sigma$ is conductivity, $v$ is velocity vector, $\varepsilon$ is permittivity and $\mu_{0}$ is magnetic

permeability.

The pulsed electromagnetic field was induced by the pulsed electrical current in the coil. As illustrated in Figure 2, the current profile in the coil is similar to a sine wave, and the discharge time is much shorter than the charging time. The relationship between the current and time is described as:

$I(t)=I_{0} \exp (-\zeta t) \sin (\omega t)$

where $I_{0}$ is a peak value in current, and $\zeta$ and $\omega$ are constant for the circuit.

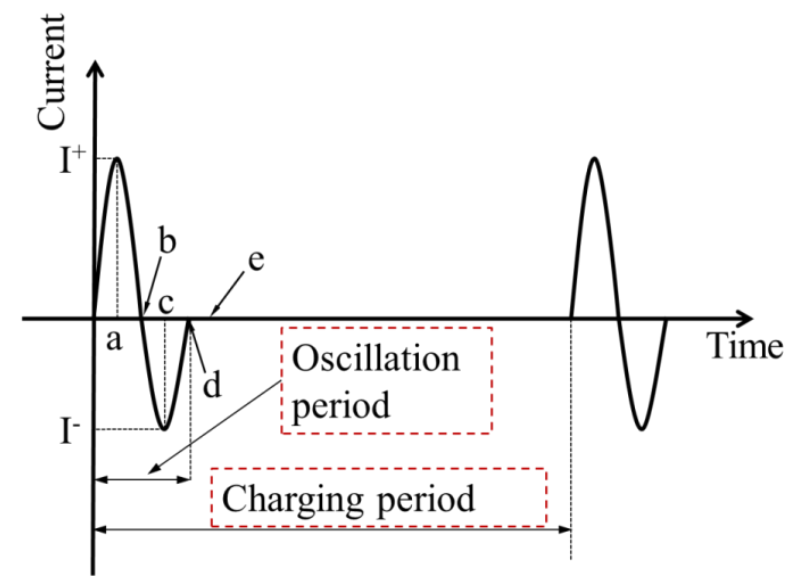

Figure 2. Variation of pulsed electricity in a coil.

a $1 / 4$ period; b $1 / 2$ period; c $3 / 4$ period; d 1 period; e $5 / 4$ periods.

In our simulation, one period was further divided into five stages for convenience.

They are stage a (T/4), b (T/2), c (3T/4), d (1T) and e (5T/4). 
The continuity and the momentum equations in two-dimensional model are described in Eq. (9) - (10):

$$
\begin{aligned}
& \frac{\partial \rho}{\partial t}+\rho \frac{\partial u_{i}}{\partial x_{i}}=0 \\
& \rho \frac{\partial\left(u_{i} u_{j}\right)}{\partial x_{j}}=-\frac{\partial P}{\partial x_{j}}+\frac{\partial}{\partial x_{j}}\left(\mu \frac{\partial u_{i}}{\partial x_{j}}\right)+\frac{\partial}{\partial x_{j}}\left(\mu \frac{\partial u_{j}}{\partial x_{j}}\right)+F
\end{aligned}
$$

where $\rho$ is density of the melt, $u_{i}$ is velocity vector components in $X$ (radial direction) and $Y$ (axil direction) directions, $P$ is pressure, and $F$ is electromagnetic force, which can be calculated according to the following equation:

$$
F=\mu_{0} J \times H
$$

Since the electromagnetic force, which induces force convection in melt, can be considered a momentum source imposed on the flow. To study this, the calculated electromagnetic force can be used in flow field simulations.

The Joule heat per unit volume produced by the electromagnetic field can be given as:

$$
\dot{q}=\int J(t)^{2} \sigma d t
$$

where $\dot{q}$ is the average value of Joule heat.

The $\dot{q}$ can be used in the temperature field simulations as follows:

$$
\frac{\partial(\rho t)}{\partial t}=\nabla \cdot\left(\frac{\lambda}{c_{p}} \nabla T\right)+\dot{q}
$$

where $\lambda$ is thermal conductivity and $c_{p}$ is specific heat. 


\section{Experimental Methods}

Commercial purity aluminum (Al: $99.7 \mathrm{wt} \%$, Fe: $0.2 \mathrm{wt} \%$, Si: $0.1 \mathrm{wt} \%$ ) was

melted in a resistance furnace. After being held for 30 minutes at $750{ }^{\circ} \mathrm{C}$, the molten melt was poured into a sand mold with an inner dimension of $\Phi 60 \mathrm{~mm} \times$ length 140 $\mathrm{mm}$ and SPMO was applied immediately. Four as-cast specimens have been produced with the same process in this study. One ingot was untreated. Three ingots were treated, which were produced with same pulsed parameters. The peak value of pulse current was $100 \mathrm{hI}$ A with a pulse frequency of $2 \mathrm{kt} \mathrm{Hz}$, which were identical to those used in numerical simulation. The as-cast specimen was cut along the central longitudinal section and then etched for metallographic examination. The etching agent was a mixture of 3 hydrochloric acid: 3 nitric acid: 5 water.

\section{Simulation results}

4.1 Distribution of the magnetic field intensity

(a)

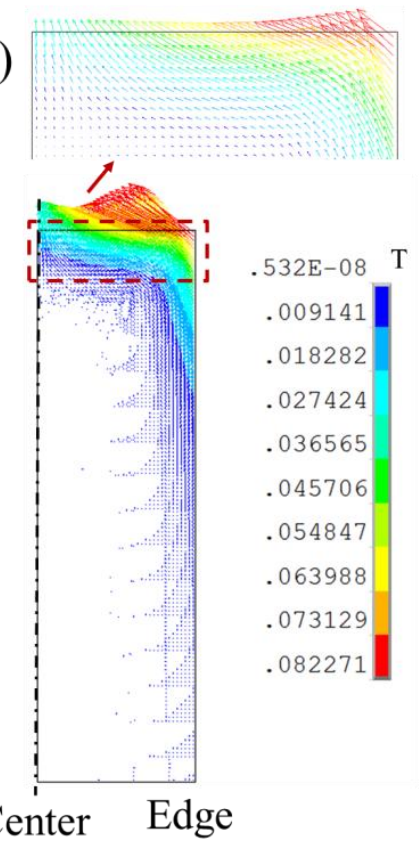

(b)

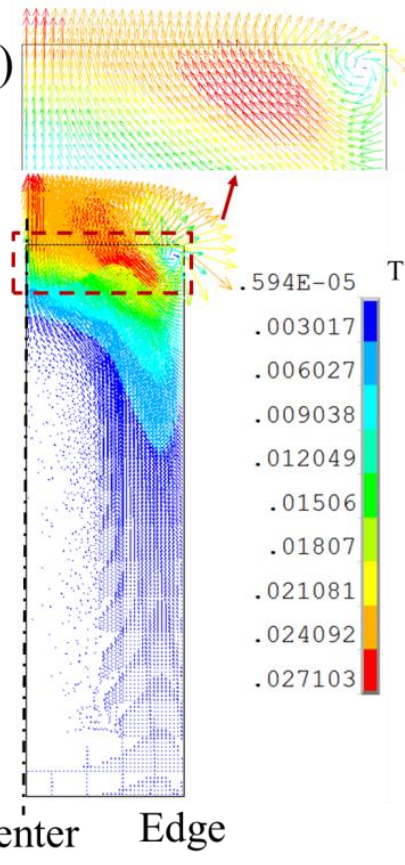

(c)

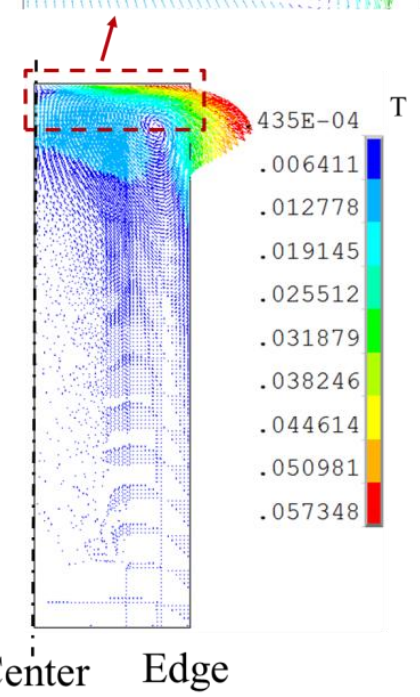


(d)
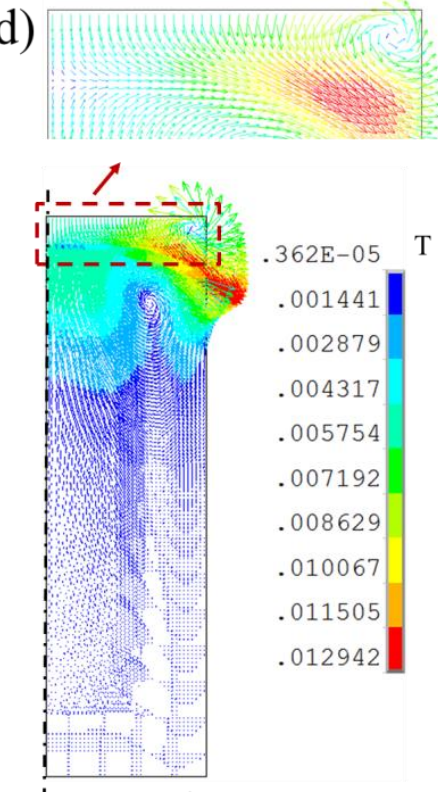

Center Edge (e)
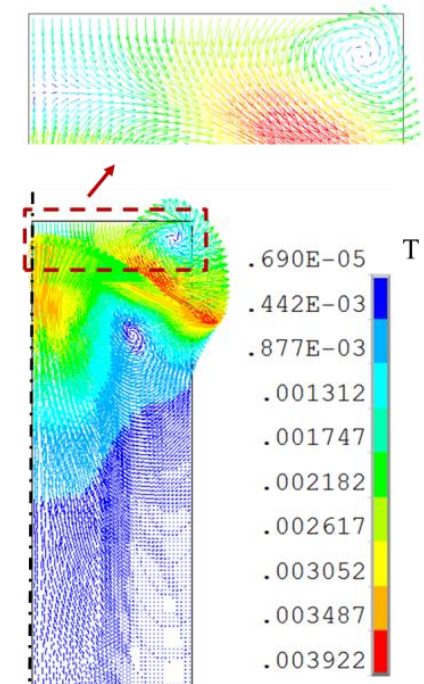

Center Edge

Figure 3. Magnetic field intensity distribution in the melt in five stages: (a) 1/4 period;

(b) 1/2 period; (c) 3/4 period; (d) 1 period; (e) 5/4 periods.

The magnetic field intensity distribution in five stages is shown in Figure 3.

Because of the skin effect of an alternating electromagnetic field, intensity decreases as the distance from the top surface increases. The maximum value of magnetic field intensity was found at the top and near the mold wall of the melt. The skin depth $\delta$ can be calculated by following formula (Jackson, 2004),

$\delta=\sqrt{2 /(\omega \mu \sigma)}$

where $\omega$ is the angular frequency of the magnetic field or eddy current, $\mu$ is the

relative magnetic permeability of the melt, $\sigma$ is the electric conductivity of the melt.

The skin depth was approximately $7 \mathrm{~mm}$ in this work. To show how magnetic field intensity changes with various durations, six nodes at different positions within skin depth (as shown in Figure 4) were selected for further study. The results are shown in 
Figure 5.

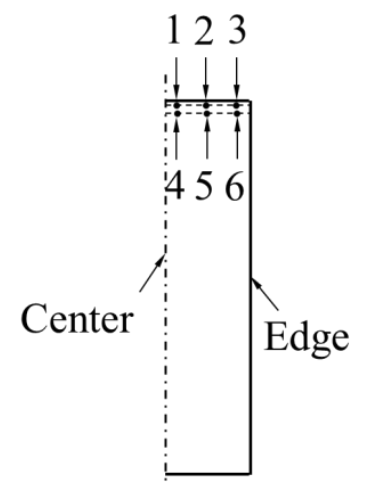

Figure 4. Schematic of nodes' position: point 1 is located at $1 \mathrm{~mm}$ from the top surface and $5 \mathrm{~mm}$ from the center, point 2 is located at $1 \mathrm{~mm}$ from the top surface and $15 \mathrm{~mm}$ from the center, point 3 is located at $1 \mathrm{~mm}$ from the top surface and $5 \mathrm{~mm}$ from the mold wall, point 4 is located at $4 \mathrm{~mm}$ from the top surface and $5 \mathrm{~mm}$ from the center, point 5 is located at $4 \mathrm{~mm}$ from the surface and $15 \mathrm{~mm}$ from the center, and point 6 is located at $4 \mathrm{~mm}$ from the top surface and $5 \mathrm{~mm}$ from the mold wall.
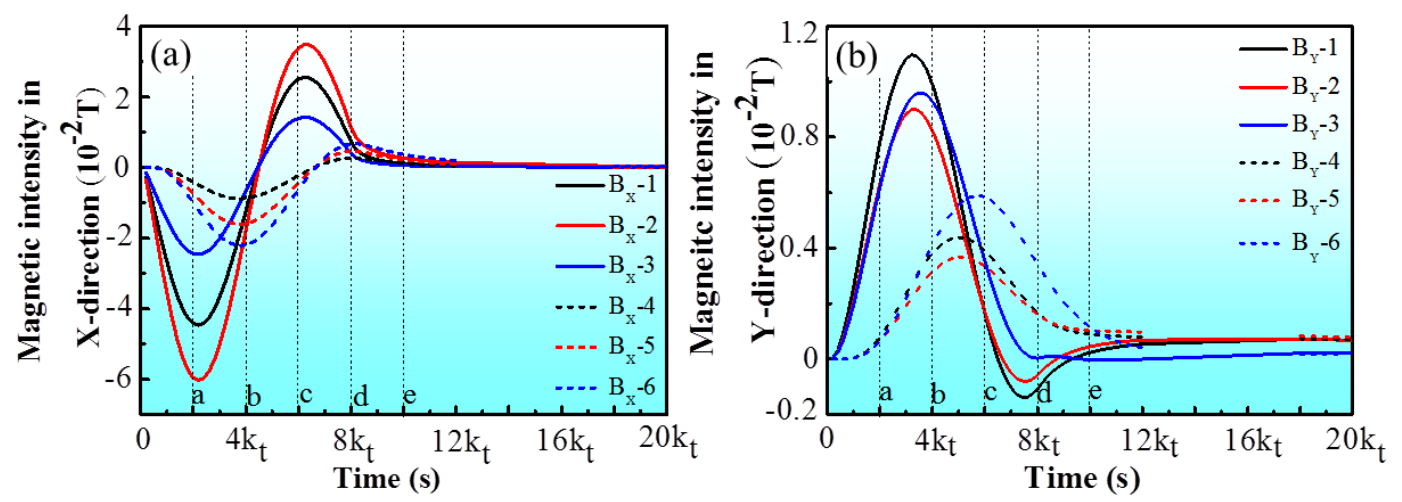

Figure 5. Magnetic field intensity vs time at different positions: (a) magnetic field intensity in radial direction; (b) magnetic field intensity in axil direction.

Comparing the curves of magnetic field intensity at different positions shows an offset time; i.e. the lag between the moment when the current increases in the coil and 
the moment the intensity of the magnetic field increases in the melt. The offset time is longer at the interior nodes (Points 4, 5, 6 in Figure 4) than at nodes near the top surface (Points 1, 2, 3 in Figure 4). This effect is evidence of propagation of the electromagnetic field (Bahmani et al., 2009). Magnetic field intensity at the top of the melt (Points 1, 2, 3 in Figure 4) is greater than that in the interior (Points 4, 5, 6 in Figure 4). This results from decay of the electromagnetic field. Magnetic field intensity can be subdivided into components associated with the $\mathrm{X}$ axis and the $\mathrm{Y}$ axis. The $\mathrm{X}$ axis refers to the horizontal direction within the ingot in Figure 1. The $\mathrm{X}$ axial component usually points from the interior to the mold wall. The $\mathrm{Y}$ axis is in the vertical direction. The Y axial component always points downward toward the mold bottom. The peak values in the two directions are different.

4.2 Distribution of the induced current

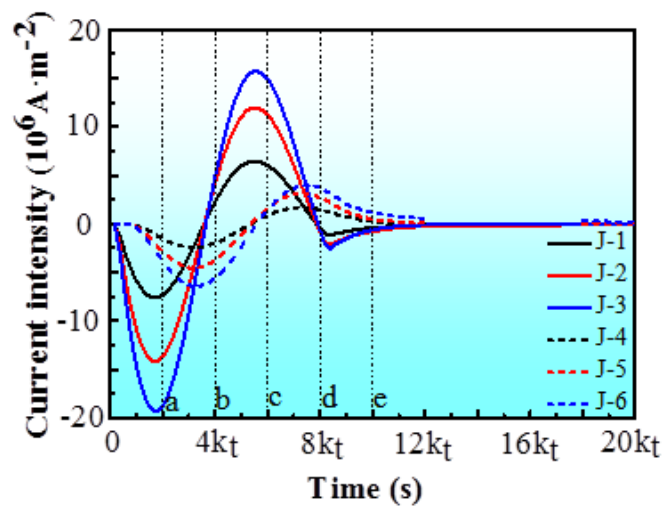

Figure 6. Induced current intensity vs time at different positions.

Figure 6 shows the variation curves of induced current at different positions in the melt. The direction of induced current is opposite to that of input. Variation of induced current intensity with time and space is similar to that of magnetic field 
intensity. Induced current is concentrated in the skin zone at the top surface. The peak value of induced current decreases as the distance from the top surface increases. In the radial direction $(\mathrm{X})$, the maximum current occurs nearest the mold wall.

4.3 Distribution of the electromagnetic force
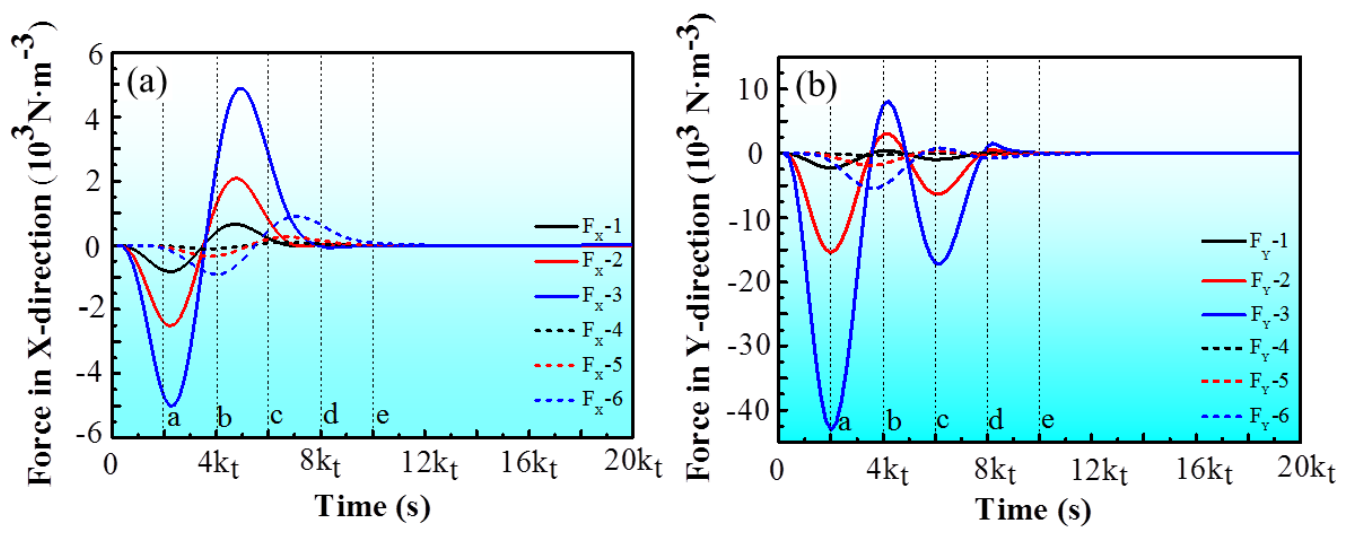

Figure 7. Electromagnetic force vs time at different positions: (a) electromagnetic force in radial direction, (b) electromagnetic force in axil direction.

Figure 7 shows the variation of electromagnetic force through whole period.

The largest electromagnetic force appears at the top surface and near the wall, with the maximum value $10^{5} \mathrm{~N} \cdot \mathrm{m}^{-3}$. In the center of the melt, electromagnetic force is nearly zero. During the whole period, compressive force thrusts through the melt in the axial direction (Y axis), reaching a peak value that is larger than that of the radial direction (X axis). The direction of electromagnetic force is different in different locations at the same time, as shown in Figure 8. 


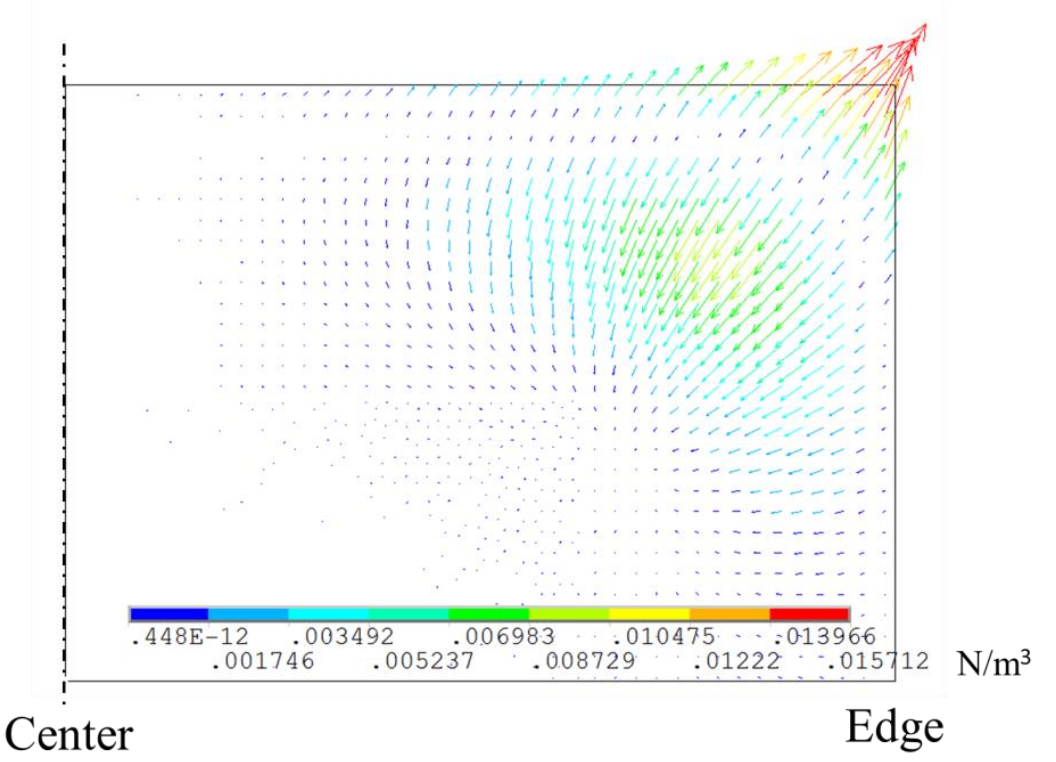

Figure 8. Local distribution of electromagnetic force in the melt at the intersection of the top surface and the mold wall.

\subsection{Flow field distribution}

Previous researchers have shown that a flow stabilized after a certain time, fluctuating in a regular pattern within the pulsed period of the magnetic field (Zhang et al., 2007). The same result was produced by our simulation. Figure 9 shows flow field distribution in the 51st pulse period, as predicted by the simulation used in this study. Electromagnetic force in the simulation mainly affects the melt in the upper part, a result that was later confirmed experimentally. Two vortexes emerge at the top surface and near to the wall. The maximum velocity reaches $7 \times 10^{-2} \mathrm{~m} \cdot \mathrm{s}^{-1}$. The electromagnetic force mentioned in Section 4.3 is about ten times that of gravity, inducing strong forced convection in the melt. 


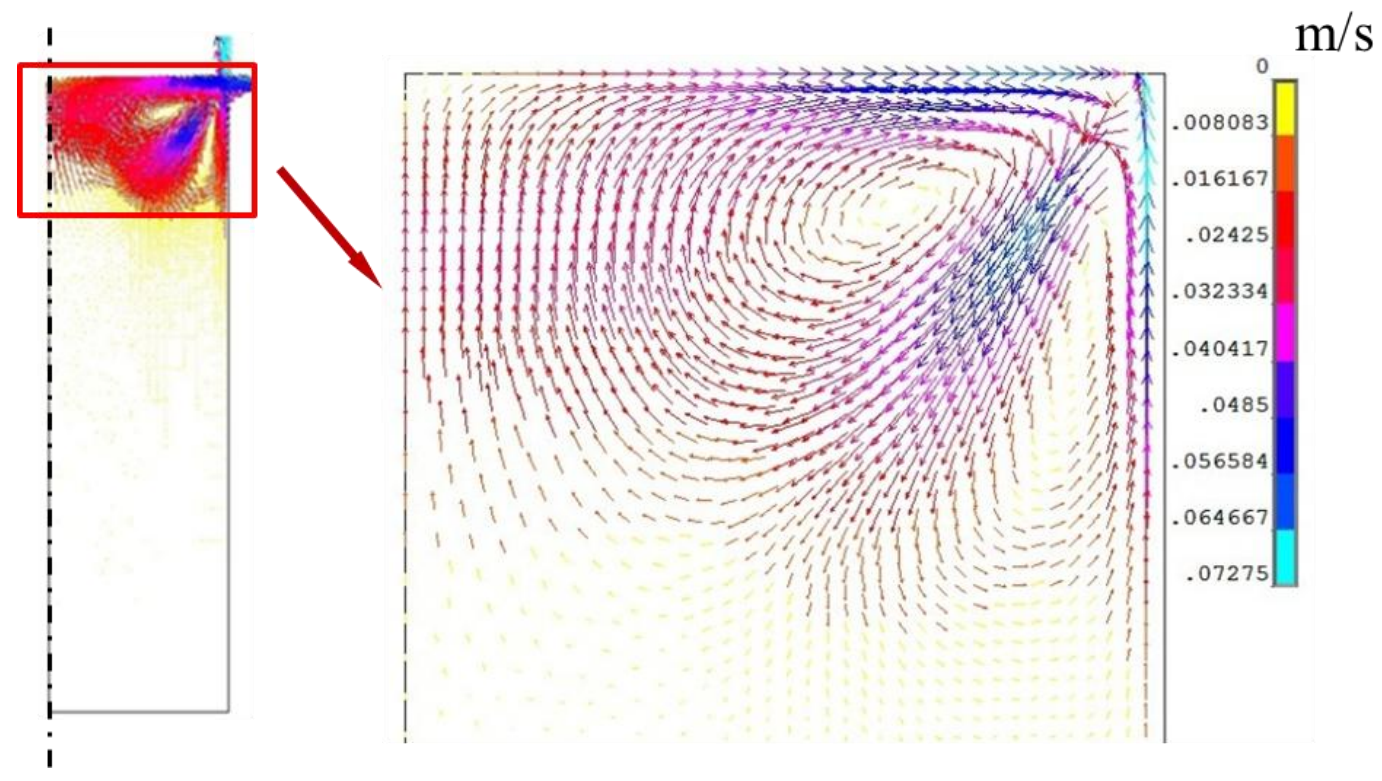

Figure 9. Flow field distribution in the 51st pulse period in the melt. The graph shown on the right is an amplification of that shown on the left.

\subsection{Temperature distribution}
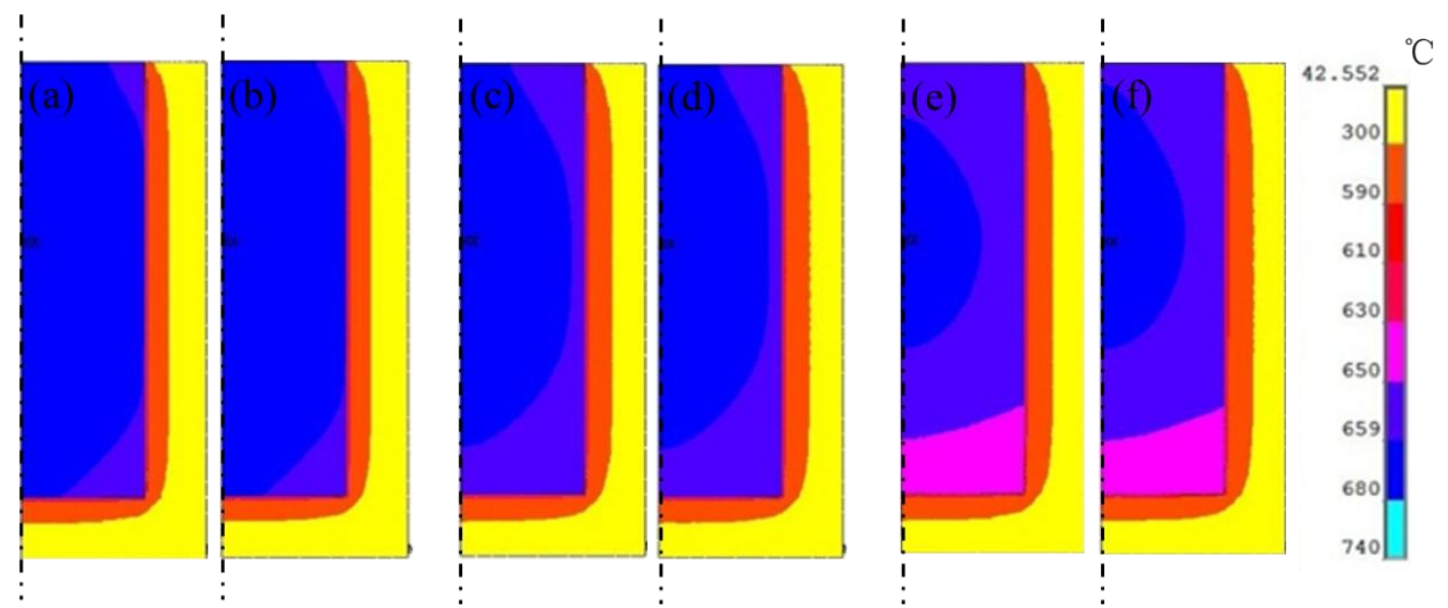

Figure 10. Comparison of temperature distribution in the melt: (a), (c), (e) show temperature distribution without SPMO at 300s, 500s, 700s; (b), (d), (f) show temperature distribution with SPMO at 300s, 500s, 700s.

Figure 10 shows temperature distribution in the melt without SPMO (Figures 10 (a), (c), (e)) and with SPMO (Figures 10 (b), (d), (f)). Temperature in the melt at a 
given position is higher with SPMO than without. This can be attributed to the additional Joule heat produced by SPMO. More additional heat is produced at the surface than in the interior of the melt. This extra heat at the surface prolongs solidification time, allowing SPMO to produce additional refined grains in the melt before a solidified shell forms on the surface (Atsumi, 1983, Qi et al., 2012).

Although surface temperature is higher with SPMO than without, undercooling is always greater at the surface and near the mold wall because of the higher cooling rates at these regions, regardless whether SPMO is used. In other words, with or without SPMO, temperature distribution remains unchanged and nucleation always occurs first at the surface and mold wall.

\section{Experimental results and discussion}

(a)

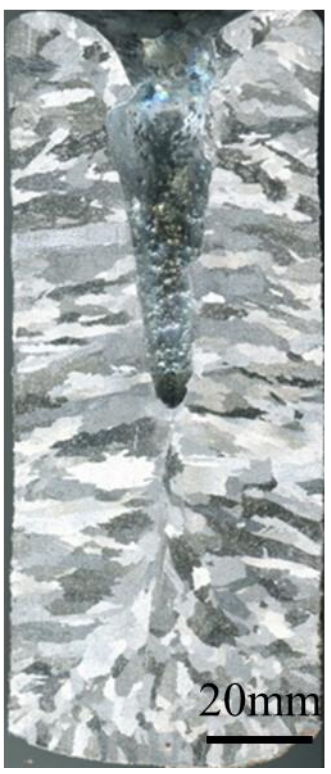

(b)

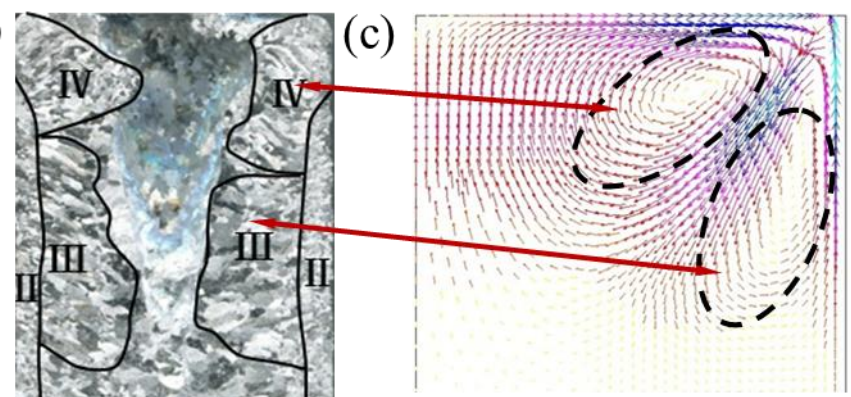

Figure11. Solidification structure of Al ingot without (a) and with (b) SPMO.

Figure 11 displays the structure of as-cast $\mathrm{Al}$ ingots treated without and with 
SPMO. Without SPMO (Figure 11 (a)), the ingot consists mainly of columnar grains with a small number of coarse equiaxial grains at the bottom of the ingot. As shown in region I of Figure 11 (b), SPMO increased the equiaxial grain zone from $19 \%$ to $60 \%$ of the ingot with finer equiaxial grains at the bottom. As the melt was expanded and compressed by tensile and compressive electromagnetic force, energy fluctuations within small spaces forced homogeneous nucleation (Li et al., 2012). Energy fluctuation in the melt can produce local overheated and undercooled regions, providing the driving force for nucleation of new grains in undercooled regions (Yan et al., 1997). Because of the larger number of nuclei, SPMO induced refinement of equiaxial grains at the bottom from $2.9 \mathrm{~mm}$ to $0.4 \mathrm{~mm}$ and columnar grains from 2.9 $\mathrm{mm}$ to $1 \mathrm{~mm}$. At the same time that SPMO causes greater homogeneity of nucleation in the interior of the melt, it also causes greater heterogeneity of nucleation at the surface. Newly formed surface nuclei then drift down into the interior. Gravity alone could not make them sink effectively; sinking is caused by the multiplier effect of electromagnetic force component associated the axil (Y) direction, which tends to thrust, in the same general direction as the force of gravity. The value of the combined force could reach up to ten times that of gravity, resulting in more nuclei drifting down into the interior of the melt. These drifted crystals, which have been called “crystallization rain" by Liao et al., 2007, create a fine equiaxial grain zone that forms at the bottom of the ingot (Part I in Figure 11 (b)). Without SPMO, refinement does not occur because fewer nuclei drift down, and each nucleus has room to grow larger 
and coarser. The simulation results showed that electromagnetic fields mainly concentrated near surface (or coil) because of skin effect. In solidification process, surface is declining continuously due to shrinkage. So if SPMO is applied in large scale ingot production, coil should move with declining surface to ensure wonderful effect.

5.2 Formation of fine equiaxial grains near the wall with SPMO

Fine equiaxial grains were developed near the mold wall in the ingot treated with SPMO, but not in the untreated ingot (Part II in Figure11 (b)). These nuclei were partially brought from the surface to the wall by the pattern of force within the electromagnetic field (Figure 11c). Because of the skin effect, the electromagnetic force is higher near the surface than in the interior of the melt. Downward flow velocity reaches maximum value at the top of the mold near the mold wall, so the drifting nuclei cannot remain there. Electromagnetic force decreases away from the surface of the melt. Once the nuclei reach a point where they encounter the optimum temperature for survival, they form a fine equiaxial grain zone (Part II in Figure11 (b), Li et al., 2011).

\subsection{Deflection of columnar grains with SPMO}

Thinner columnar grains were found in the SPMO treated ingot. Their growth direction was not perpendicular to the mold wall as in the untreated ingot but deflected either to the top (Part IV in Figure 11 (b)) or the bottom (Part III in Figure 11 (b)). The natural convection caused by the temperature and gravity was very weak 
because size of the ingot was small. Therefore, the natural convection had little effect on the columnar grains growth. So the growth direction of columnar grains in the ingot made without SPMO was related mainly to the direction of heat flux which is almost perpendicular to the mold wall. But with SPMO, the flow pattern changed with the electromagnetic force.

Forced convection induced by electromagnetic force is known to cause columnar crystal growth (Jeong et al., 2003). In SPMO treated ingot the flow pattern changes with the electromagnetic force and creates two vortexes (Figure 11 (c)). Our observed boundary between upward columnar and downward columnar grains was consistent with the boundary between the two vortexes (cf: Figure 11(b) Part III and downward vortex in Figure 11(c); Part IV in Figure 11(b) and upward vortex in Figure 11(c)). Moreover, the angle of the columnar grains deflecting upward (Part IV in Figure 11 (b)) was greater than that of those deflecting downward (Part III in Figure 11 (b)), which indicates that the electromagnetic force was greater at the surface of the melt than in the interior (Figure 7). In other words, the closer the surface of the melt, the higher the electromagnetic force and therefore the velocity of the fluid, and the greater the deflection of columnar grain direction. The difference between the experimental and numerical simulation results was assessed, which was about 7 8\%.

The same changing tendency was found in another two treated ingots in the experiment with the same parameters as shown in Figure 12. And the characteristic of SPMO treated ingots could be concluded in our past experiment results with different 
parameters (Yin et al., 2012).

(a)

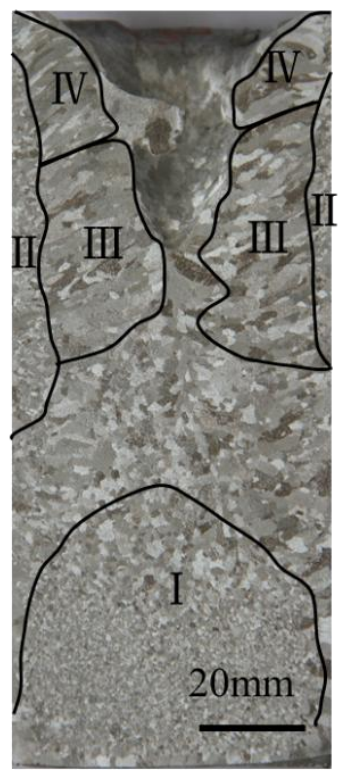

(b)

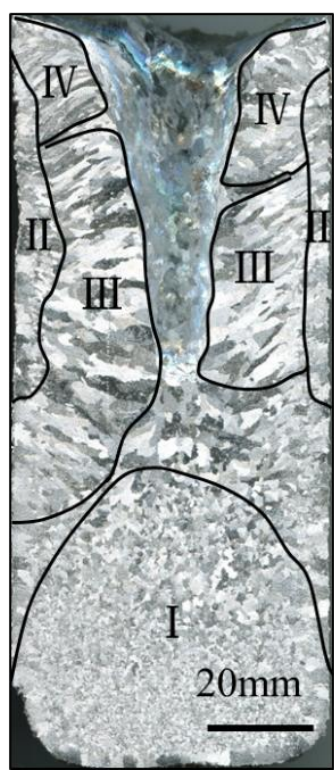

Figure12. Solidification structure of two SPMO treated ingots.

\section{Conclusions}

1) Numerical simulation was used to illustrate the influence of SPMO on the solidification process, which was able to predict and explain the solidification structure achieved in an ingot formed by SPMO.

2) The electromagnetic force and Joule heat produced by SPMO mainly concentrated at the top of the melt. Electromagnetic force provided enough power to cause the nuclei that were created on the surface to fall and drift down into the melt. Joule heat prolonged the solidification time of the surface layer of the melt.

3) The forced convection induced by electromagnetic force reduced the temperature gradient in most of the areas and changed the growth direction of the columnar grains.

\section{Acknowledgements}

Work supported by the research grant (No.14DZ2261200) from Science and 
Technology Commission of Shanghai Municipality. The authors gratefully

acknowledge financial support from the National Basic Research Program of China (973 program, Granted No.2011CB012902), USNSF (Cooperative Agreement DMR0654118 and 1157490), the State of Florida and the US Department of Energy. We thank Dr. Tyler for editing the manuscript.

\section{References}

Atsumi, O., 1983. Metal Solidification. Tang, Y.B., Zhang, Z.D., transl. Beijing: Mechanical Industry Press, 113-115.

Gao, Y.L., Li, Q.S., Gong, Y.Y., Zhai, Q.J., 2007. Comparative study on structural transformation of low-melting stainless steel under external pulse magnetic field. Materials Letters 61, 4011-4014.

Gong, Y.Y., Luo, J, Jing J.X., Xia, Z.Q., Zhai, Q.J., 2008. Structure refinement of pure aluminum by pulse magneto-oscillation. Materials Science and Engineering A 497, $147-152$.

Ha, M.Y., Lee, H.G., Seong, S.H., 2003. Numerical simulation of three-dimensional flow, heat transfer, and solidification of steel in continuous casting mold with electromagnetic brake. Journal of Materials Processing Technology 133, 322-339.

Huang, J., Wang, B.F., Zhao, L.P., Li, J.C., 2008. Numerical simulation of magnetic field and flow field in aluminum melt with electromagnetic Stirring. Special Casting \& Nonferrous Alloys, (2): 119-121. (In Chinese)

Jackson, J.D., 2004. Classical Electrodynamics, third ed. Higher Education Press, 
219-220.

Jeong, J.H., Dantzig, J.A., Goldenfeld, N., 2003. Dendritic growth with fluid flow in pure materials. Metallurgical and Materials Transactions A 34, 459-466.

Kermanpur, A., Jafari, M., Vaghayenegar, M., 2011. Electromagnetic-thermal coupled simulation of levitation melting of metals. Journal of Materials Processing Technology. 211, 222-229.

Kolesnichenko, A.F., Podoltsev, A.D., Kucheryavaya, I.N., 1994. Action of pulse magnetic field on molten metal. ISIJ International 34, 715-721.

Le, Q.S., Guo, S.J., Zhao, Z.H., Cui, J.Z., Zhang, X.J., 2007. Numerical simulation of electromagnetic DC casting of magnesium alloys. Journal of Materials Processing Technology. 183, 194-201.

Li, B., Yin, Z.X., Gong, Y.Y., Li, K.F., Zheng, H.X., Li, R.X., Zhai, Q.J., 2011. Effect of temperature field on solidification structure of pure Al under pulse magneto-oscillation. China Foundry 8, 172-176.

Li, Q.S., Li, H.B., Zhai, Q.J., 2006. Structure evolution and solidification behavior of austenitic stainless steel in pulsed magnetic field. Journal of Iron and Steel Research $13,69-72$

Li, Y.J., Ma, X.P., Yang, Y.S., 2011. Grain refinement of as-cast superalloy IN718 under action of low voltage pulsed magnetic field. Trans. Nonferrous Met. Soc. China $21,1277-1282$.

Li, Y.J., Tao, W.Z., Yang, Y.S., 2012. Grain refinement of Al-Cu alloy in low voltage 
pulsed magnetic field. Journal of Materials Processing Technology 212, 903-909.

Liao, X.L., Zhai, Q.J., Luo, J., Chen, W.J., Gong, Y.Y., 2007. Refining mechanism of the electric current pulse on the solidification structure of pure aluminum. Acta Materialia 55, 2103-3109.

Ma, X.P., Yang, Y.S., Wang, B., 2009. Effect of pulsed magnetic field on superalloy melt. International Journal of Heat and Mass Transfer 52, 5285-5292.

Paul P.U., 2004, College physics (Photocopied edition). Beijing: China Machine Press, 485.

Puga, H., Costa S., Barbosa, J., Ribeiro, S., Prokic, M., 2011, Influence of ultrasonic melt treatment on microstructure and mechanical properties of $\mathrm{AlSi} 9 \mathrm{Cu} 3$ alloy. Journal of Material Processing Technology 211, 1729-1735.

Qi, J.G., Dai, S., Zhao, Z.F., Zhang, D.J., Wang, J.Z., Cang, D.Q., 2012. Model of solidification characteristics of $\mathrm{Cu}-\mathrm{Al}$ alloy modified by electric pulse. The Chinese Journal of Nonferrous Metals 22, 224-229.

Yan, H.C., He, G.H., Zhou, B.L., Qin, R.S., Guo, J.D., Shen, Y.F., 1997. The influence of pulse electric discharging on solidified structure of $\mathrm{Sn}-10 \% \mathrm{~Pb}$ alloy. Acta metallurgica sinica 33, 352-358.

Yin, Z.X., Gong, Y.Y., Li, B., Cheng, Y.F., Liang, D., Zhai, Q.J., 2012. Refining of pure aluminum cast structure by surface magneto-oscillation. Journal of Materials Processing Technology 212, 2629-2634.

Yin, Z.X., 2012. Effect of Electric Current Pulse on As-cast Structure of Metals and 
Alloys under Three Introduction Ways. Shanghai University Doctoral Dissertation, 108-109.

Zhang, H.T., Nagaumi, H., Cui, J.Z., 2007. Coupled modeling of electromagnetic field, fluid flow, heat transfer and solidification during low frequency electromagnetic casting of 7XXX aluminum alloys Part II : The effects of electromagnetic parameters on casting processes. Materials Science and Engineering A 448, 177-188.

Zhang, Q., Jin, J.Z., Wang, T.M., Li, T.J., Guo, Q.T., 2007. Analysis of molten metal flow in rotating magnetic field. The Chinese Journal of Nonferrous Metals 17, 98-104.

Zhao, Z.H., Cui, J.Z., Dong, J., Zhang, B.J., 2007. Effect of low-frequency magnetic field on microstructures and macrosegregation of horizontal direct chill casting 7075 aluminum alloy. Journal of Materials Processing Technology. 182, 185-190.

Zhao, Z.L., Liu, Y., Liu, L., 2011. Grain refinement induced by a pulse magnetic field and synchronous solidification. Materials and Manufacturing Processes 26, 1202-1206. 


\section{Figure captions:}

Figure 1 Schematic of SPMO casting setup (a) and FEM model used in the simulation (b).

Figure 2 Variation of pulsed electricity in a coil.

Figure 3 Magnetic field intensity distribution in the melt in five stages: (a) 1/4 period;

(b) 1/2 period; (c) 3/4 period; (d) 1 period; (e) 5/4 periods.

Figure 4 Schematic of nodes' position: point 1 is located at $1 \mathrm{~mm}$ from the top surface and $5 \mathrm{~mm}$ from the center, point 2 is located at $1 \mathrm{~mm}$ from the top surface and $15 \mathrm{~mm}$ from the center, point 3 is located at $1 \mathrm{~mm}$ from the top surface and $5 \mathrm{~mm}$ from the mold wall, point 4 is located at $4 \mathrm{~mm}$ from the top surface and $5 \mathrm{~mm}$ from the center, point 5 is located at $4 \mathrm{~mm}$ from the surface and $15 \mathrm{~mm}$ from the center, and point 6 is located at $4 \mathrm{~mm}$ from the top surface and $5 \mathrm{~mm}$ from the mold wall.

Figure 5 Magnetic field intensity vs time at different positions: (a) magnetic field intensity in radial direction; (b) magnetic field intensity in axil direction.

Figure 6 Induced current intensity vs time at different positions.

Figure 7 Electromagnetic force vs time at different positions: (a) electromagnetic 
force in radial direction, (b) electromagnetic force in axil direction.

Figure 8 Local distribution of electromagnetic force in the melt at the intersection of the top surface and the mold wall.

Figure 9 Flow field distribution in the 51st pulse period in the melt. The graph shown on the right is an amplification of that shown on the left.

Figure 10 Comparison of temperature distribution in the melt: (a), (c), (e) show temperature distribution without SPMO at 300s, 500s, 700s; (b), (d), (f) show temperature distribution with SPMO at 300s, 500s, 700s.

Figure 11 Solidification structure of Al ingot without (a) and with (b) SPMO.

Figure 12 Solidification structure of two SPMO treated ingots

\section{Nomenclature}

$B$ magnetic flux density vector $\left[\mathrm{Wb} \mathrm{m}^{-2}\right]$

$C_{p}$ specific heat $\left[\mathrm{J} \mathrm{kg}^{-1} \mathrm{~K}^{-1}\right]$

$D$ electric flux density vector $\left[\mathrm{C} \mathrm{s}^{-2}\right]$

$E$ electric field intensity vector $\left[\mathrm{V} \mathrm{m}^{-1}\right]$

$F$ electromagnetic force $[\mathrm{N}]$

$H$ magnetic field intensity vector $\left[\mathrm{A} \mathrm{m}^{-1}\right.$ ]

$J$ total current density vector $\left[\mathrm{A} \mathrm{m}^{-2}\right.$ ]

$P$ pressure $[\mathrm{Pa}]$

$Q_{v}$ volumetric heat source $\left[\mathrm{J} \mathrm{m}^{-3} \mathrm{~s}^{-1}\right.$ ]

$T$ temperature $\left[{ }^{\circ} \mathrm{C}\right]$ 
$t$ time [s]

$U_{i}$ orthogonal velocity $\left[\mathrm{m} \mathrm{s}^{-1}\right]$

$X_{i}$ global Cartesian coordinates

$\varepsilon$ permittivity $\left[\mathrm{F} \mathrm{m}^{-1}\right]$

$\lambda$ second coefficient of viscosity $\left[\mathrm{kg} \mathrm{m}^{-1} \mathrm{~s}^{-1}\right]$

$\mu_{0}$ magnetic permeability $\left[\mathrm{H} \mathrm{m}^{-1}\right]$

$\rho_{0}$ electric charge density $\left[\mathrm{C} \mathrm{m}^{-3}\right]$

$\sigma$ conductivity $\left[\Omega^{-1} \mathrm{~m}^{-1}\right]$

I electric current

$\zeta \omega$ constant 